\title{
Ergodic Matrix and Hybrid-key Based Image Cryptosystem
}

\author{
Xiaoyi Zhou ${ }^{* 1,2}$, Jixin $\mathrm{Ma}^{2}$ \\ 2. School of Computing \& Mathematical Sciences, University of Greenwich, London, UK \\ Email: \{zx09, J.ma\}@gre.ac.uk \\ Wencai $\mathrm{Du}^{1}$ \\ 1. Info. Science \& Tech.School, Hainan University, Haikou, Hainan, China \\ Email: georgewend@gmail.com \\ Yongzhe Zhao ${ }^{* 3}$ \\ 3. Computer Science and Technology School, Jilin University, Changchun, Jilin, China \\ yongzhe@jlu.edu.cn \\ * Corresponding Author
}

\begin{abstract}
The existing traditional cryptosystems, such as RSA, DES, IDEA, SAFER and FEAL, are not ideal for image encryption because of their slow speed and ineffectiveness in removing the correlations of the adjacent pixels. Meanwhile chaos-based cryptosystems, which have been extensively used over the past two decades, are almost all based on symmetric cryptography. Symmetric cryptography is much faster than asymmetric ciphers, but the requirements for key exchange make them hard to use. To remedy this imperfection, a hybrid-key based image encryption and authentication scheme is proposed in this paper. In particular, ergodic matrices are utilized not only as public keys throughout the encryption/decryption process, but also as essential parameters in the confusion and diffusion stages. The experimental results, statistical analysis and sensitivity-based tests confirm that, compared to the existing chaos-based cryptosystems, the proposed image encryption scheme provides a more secure means of image encryption and transmission.
\end{abstract}

Index Terms- hybrid-key, ergodic matrix, symmetric, asymmetric, entropy, diffusion

\section{INTRODUCTION}

Transmitting information through the internet has been a mainstream task in modern life for these reasons: geographical position independence, fast speeds, and low costs. However, while we are taking the advantage of the internet, unauthorized individuals can purloin the images for processing, distributing or replicating. Hence information security is an important problem. Therefore cryptography plays a vital role in many fields, such as mobile phone communications, sending private emails, security of bank cards, online payments, users' passwords, online personal photograph albums and other touches of our daily lives [1]. Cryptography is a technique of transforming plaintexts into ciphertexts and retransforming the messages back to the original form. Modern cryptography involves not only the disciplines of mathematics but also computer sciences and engineering.

Image cryptography has various applications in telemedicine, military image database and multimedia Copyright (C) 2011 MECS systems, etc. Although there are many cryptosystems, such as RSA, DES, IDEA, SAFER and FEAL, which can be used to encrypt images, these are not ideal for two reasons [2]. One is that the image size is generally much greater than that of text. This results in conventional cryptosystems taking much more time to encrypt images directly. The other reason is that image data has high correlation among adjacent pixels. Consequently, it is rather difficult for these cryptosystems to shuffle and diffuse image data effectively.

By and large, there are two main schemes that can be used to protect digital images:

(1) Information hiding, which is a technology that uses anonymity, watermarking, covert channel and steganography; and

(2) Encryption, which includes traditional encryption and other such as chaotic encryption [3-4].

Chaos-based cryptosystems usually have higher speeds and lower costs. Moreover, these systems are sensitive to initial conditions and control parameters. These optimistic characters make them suitable for image encryption. In this respect, during the past decade a great number of chaotic systems have been proposed. For example, Chen et al. used a 3D baker map [6] and a 3D cat map [7] in the permutation process. Guan et al. employed a 2D cat map for substitution and the diffusion of Chen's chaotic system for masking the pixel values [8]. Jiri Giesl et al. used the chaotic maps of Peter de Jong's attractor to improve the chaos image encryption speed [5]. Yang et al. introduced a keyed hash function to generate a 128-bit hash value so that the scheme could be used to encrypt and authenticate [9].

However, the deficiencies of these chaos-based cryptosystems can be summarized as below:

(1) The communication session is mostly based on symmetric cryptography.

(2) Lack of authentication, which means it is difficult for the receiver to confirm that the cipher image is sent by the one he wants to communicate with.

The disadvantage of symmetric cryptography is that it 
presumes two parties have agreed on a key and been able to exchange that key in a secure manner prior to communication. Since the cipher key is public, the distribution and management of keys are much simpler, i.e. in a population of $n$ people the total keys are $4 n$. For this reason, the requirements for key exchange make symmetric cryptosystems difficult to use. In addition, the distribution of keys remains the biggest challenge in the use of cryptosystems of this kind [12].

Asymmetric (also called public-key) cipher is noticed as the most significant new development in cryptography in the last 30-40 years [25]. It is a technique employed by many cryptographic algorithms and cryptosystems. Its feature lies in the asymmetric key algorithm, where the key used to encrypt a plain-text is different from the key used to decrypt it. Each user holds a pair of cryptographic keys - public keys and private keys [21-22]. The public keys can be distributed whilst the private key is kept secret. Plain-texts are encrypted with the recipient's public key and can be uniquely decrypted with the private key held by the recipient. This thought came from the publication of "New Directions in Cryptography" by Diffie and Hellman in 1976.

However, in practice, the asymmetric-key cryptography system has not replaced the symmetric-key cryptography system. Though the asymmetric-key encryption system is based on sophisticated mathematical problems (its calculation is very complicated and makes it more secure), its speed is also far slower than symmetric-key encryption systems. As a result, in practical applications we can make use of the advantages of both these two algorithms, using symmetric algorithm to encrypt files and asymmetric algorithm to encrypt the keys of the encrypted document key (or it may be called session key), which is a hybrid encryption system. This provides a better way to solve the computing speed issues and the key distribution and management issues.

To realize the hybrid image cryptosystem, ergodic matrix shall be used in this paper. Zhao et al. [10-11] have proved some theorems of ergodic matrix; it is said that the matrix has large period over $\mathbb{F}^{q}$. This profound property makes it feasible in the image encryption process.

The rest of this paper is organized as follows: Section 2 gives the background about ergodic matrices; Section 3 is devoted to the description of the proposed image encryption and authentication scheme; the security of this scheme is tested in Section 4; Section 5 analyzes the performances; and finally, some conclusions and future works are drawn in Section 6.

\section{OVERVIEW OF ERGODIC MATRIX}

Ergodic matrix was introduced by Zhao et al. [10-11]. The basic idea can be briefly described as below:

Let $\mathbb{F}_{n \times n}^{q}$ be a set of all $n \times n$ matrices over the finite field $\mathbb{F}^{q},\left(\mathbb{F}_{n \times n}^{q},+, \times\right)$ form a 1-ring, here + and $\times$ are addition and multiplication over $\mathbb{F}^{q}$, respectively. We randomly generate two nonsingular matrices $Q_{1}, Q_{2} \in$ $\mathbb{F}_{n \times n}^{q}$, then:
(1) $\left(\mathbb{F}_{n \times n}^{q}, \times\right)$ is a monoid, its identity element is $I_{n \times n}$,

(2) $\left(\left\langle Q_{1}\right\rangle, \times\right)$ and $\left(\left\langle Q_{2}\right\rangle, \times\right)$ are Abelian groups, their identity elements are also $I_{n \times n}$. Here $Q_{1}, Q_{2}$ are nonsingular and $Q_{1}, Q_{2} \in \mathbb{F}_{n \times n}^{q}$, and

(3) for any $m_{1}, m_{2} \in \mathbb{F}_{n \times n}^{q}$, generally $m_{1} \times m_{2} \neq m_{2} \times m_{1}$. i.e. the multiplication is not commutative in $\mathbb{F}_{n \times n}^{q}$.

Ergodic matrix has the following definitions and properties:

Definition 1: Given $Q \in \mathbb{F}_{n \times n}^{q}$ if $\forall v \in \mathbb{F}_{n \times 1}^{q} \backslash\{\boldsymbol{0}\}$, $\left\{Q v, Q^{2} v, \ldots, Q^{q^{n}-1} v\right\}$ just takes over $\mathbb{F}_{n \times 1}^{q} \backslash\{\boldsymbol{0}\}$, then $Q$ is what so-called ergodic matrix over finite field $\mathbb{F}^{q}$. $\left(\right.$ Here $\mathbf{0}=\left[\begin{array}{llll}0 & 0 & \ldots & 0\end{array}\right]^{\mathrm{T}}$ )

Definition 2: Given $Q \in \mathbb{F}_{n \times n}^{q}$, if $\langle Q\rangle=\left\{Q^{x} \mid x=1,2\right.$, $3, \ldots\}$, then $\langle Q\rangle$ is the generating set of $Q$ over $\mathbb{F}_{n \times n}^{q}$.

Theorem 1: $Q \in \mathbb{F}_{n \times n}^{q}$ is an ergodic matrix if and only if the order of $Q$ is $\left(q^{n}-1\right)$ after the multiplication of $Q$ over finite field $\mathrm{F}^{q}$.

Theorem 2: Given $n$ and $\left(q^{n}-1\right)$ are coprime, $g=\left(g_{n} \cdots\right.$ $\left.g_{2} g_{1}\right) \in\left(\mathbb{F}^{q}\right)^{n} \wedge\left(g_{n} \neq 0\right)$, given $Q$ is an ergodic matrix, then $\left(\mathbb{F}^{q}[Q],+, \bullet\right)$ constructs a field of $q^{n}$ elements, $\left\{Q^{0}=I\right.$, $\left.Q^{1}, \cdots, Q^{n-1}\right\}$ is a basis of $\mathbb{F}^{q}[Q]$ over $\mathbb{F}^{q}$.

Lemma 1: For $\forall v \in \mathbb{F}^{q}[Q]$, there is unique $a_{0}, a_{1}, \ldots$, $a_{\mathrm{n}-1} \in \mathbb{F}^{q}$ such that $v=a_{0} I+a_{1} Q+a_{2} Q^{2}+\ldots+a_{\mathrm{n}-1} Q^{n-1}$.

Theorem 3: Given $Q \in \mathbb{F}_{n \times n}^{q}$ is an ergodic matrix, $Q^{\mathrm{T}}$ must be an ergodic matrix as well.

Theorem 4: Given $Q \in \mathbb{F}_{n \times n}^{q}$ is an ergodic matrix, then for $\forall v \in \mathbb{F}_{n \times 1}^{q} \backslash\{\boldsymbol{0}\},\left\{v^{\mathrm{T}} Q, v^{\mathrm{T}} Q^{2}, \ldots, v^{\mathrm{T}} Q^{q^{n}-1}\right\}$ just takes over $\left\{v^{T} \mid v \in \mathbb{F}_{n \times 1}^{q}\right\} \backslash\left\{0^{\mathrm{T}}\right\}$.

Theorem 5: Given $Q \in \mathbb{F}_{n \times n}^{q}$ is an ergodic matrix, there are $\varphi\left(q^{n}-1\right)$ ergodic matrices in $\langle Q\rangle(\varphi(x)$ is Euler function). These ergodic matrices have the same generation set.

Definition 3: Ergodic matrices are equivalent if they have the same generation set.

From the theorems above, over finite field $\mathbb{F}^{q}$, all $n \times n$ ergodic matrices have the same order and their generating sets have the same size, which are larger than that of any other $n \times n$ non-ergodic matrices. Take a random ergodic matrix $Q \in \mathbb{F}_{50 \times 50}^{256}$ as an example, then the image of the matrix and the histogram is shown in Fig. 1.

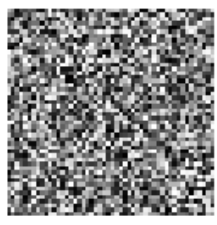

(a)

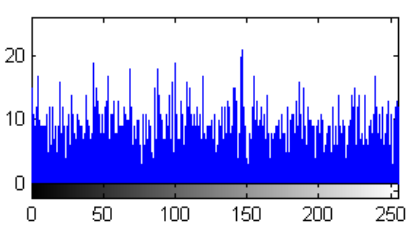

(b)
Figure 1: The image of a random ergodic matrix $Q \in \mathbb{F}_{50 \times 50}^{256}$ and the corresponding histogram (a) Image of random ergodic matrix, (b) Histogram of the corresponding ergodic matrix

This figure implies that an ergodic matrix is almost uniformly distributed, thus it can be used to encrypt an image.

\section{THE PROPOSED IMAGE ENCRYPTION ALGORITHM}


Fridrich suggested that an image encryption scheme should be composed of confusion and diffusion [4]. The confusion process permutes all the pixels without changing their values, which make it difficult to resist statistical attacks. Then in the diffusion process, the pixel values are modified such that a tiny change, for instance, the value of one pixel increased by only 1 bit, will spread out to all pixels in the cipher-image.

\section{A. Communication process}

The communication process is composed of encryption, decryption and authentication.

In consideration of the basic need of cryptology, the cipher-text should have close connection to the key. There are two ways to realize this requirement [20]: one is to utilize a good key generation mechanism, another is to thoroughly mix the key with the plain-text in the encryption process.

Therefore, to realize the hybrid-based cryptosystem that satisfies the requirement, we carry out the process as below:

(1) Alice and Bob respectively take $\left(x_{\mathrm{a}}, y_{\mathrm{a}}=\boldsymbol{f}\left(x_{\mathrm{a}}, k_{\mathrm{a}}\right)\right)$ and $\left(x_{\mathrm{b}}, y_{\mathrm{b}}=\boldsymbol{f}\left(x_{\mathrm{b}}, k_{\mathrm{b}}\right)\right)$ as public-keys, $k_{\mathrm{a}}$ and $k_{\mathrm{b}}$ as privatekeys. Here $x_{\mathrm{a}}=Q_{\mathrm{a}}, y_{\mathrm{a}}=\boldsymbol{f}\left(x_{\mathrm{a}}, k_{\mathrm{a}}\right)=Q_{\mathrm{a}}{ }^{k_{a}} ; x_{\mathrm{b}}=Q_{\mathrm{b}}, y_{\mathrm{b}}=\boldsymbol{f}\left(x_{\mathrm{b}}, k_{\mathrm{b}}\right)$ $=Q_{\mathrm{b}}{ }^{{ }_{b}}$.

(2) When Alice wants to communicate with Bob, she generates a random key $k_{x} \in \boldsymbol{K}$ and computes $K_{1}=\boldsymbol{f}\left(x_{\mathrm{b}}, k_{x}\right)=Q_{\mathrm{b}}{ }^{k_{x}}, K_{\mathrm{a}}=\boldsymbol{f}\left(y_{\mathrm{b}}, k_{x}\right)=Q_{\mathrm{b}}{ }^{k_{b} k_{x}}$. Then she gets $C I D_{\mathrm{a}}$ by encrypting her identity $I D_{\mathrm{a}}$ with $K_{1}$. For example, she encodes her identity information into an $n$-order matrix, then XOR with $K_{1}$, or gets $C I D_{\mathrm{a}}$ by $K_{1} \times I D_{\mathrm{a}}=Q_{\mathrm{b}}{ }^{k x} I D_{\mathrm{a}}$. After this, she sends $\left(C I D_{\mathrm{a}}, K_{\mathrm{a}}\right)$ to Bob.

(3) Bob uses his own key $k_{\mathrm{b}}$ to deduce $K_{1}=\boldsymbol{f}\left(K_{\mathrm{a}}\right.$,$\left.k_{\mathrm{b}}\right)=\left(Q_{\mathrm{b}}{ }^{k_{b} k_{x}}\right) Q_{\mathrm{b}}{ }^{-k_{b}}=Q_{\mathrm{b}}{ }^{k_{x}}$ and decipher $I D_{\mathrm{a}}$ by XOR $C I D_{\mathrm{a}}$ with $Q_{\mathrm{b}}{ }^{k_{x}}$, or by the equation $I D_{\mathrm{a}}=K_{1}{ }^{-1} \times C I D_{\mathrm{a}}$, thus he gets Alice's public-key $\left(x_{\mathrm{a}}, y_{\mathrm{a}}\right)$ and makes sure $C I D_{\mathrm{a}}$ is actually from Alice.

(4) Bob generates a random key $k_{y} \in \boldsymbol{K}$, and computes $K_{2}=\boldsymbol{f}\left(x_{\mathrm{a}}, k_{y}\right)=Q_{\mathrm{a}}{ }^{k_{y}}, K_{\mathrm{b}}=\boldsymbol{f}\left(y_{\mathrm{a}}, k_{\mathrm{y}}\right)=Q_{\mathrm{a}}{ }^{k_{a} k_{y}}, C K_{\mathrm{b}}=\boldsymbol{f}\left(K_{1}\right.$, $\left.K_{\mathrm{b}}\right)=Q_{\mathrm{b}}{ }^{k_{x}} Q_{\mathrm{a}}{ }^{k_{a} k_{y}}$. Then he sends $C K_{\mathrm{b}}$ to Alice.

(5) Alice decrypts $K_{\mathrm{b}}$ and $K_{2}$ by $K_{\mathrm{b}}=\boldsymbol{f}^{-1}\left(C K_{\mathrm{b}}, K_{1}\right)$, $K_{2}=\boldsymbol{f}^{l}\left(K_{\mathrm{b}}, s k_{\mathrm{a}}\right)$. Thus $K_{1}$ and $K_{2}$ can be used as session keys between Alice and Bob.

(6) If Alice wants to secretly send an image to Bob, she may encrypt the image by one of the session keys. Take $K_{1}$ for example, then she gets the cipher-image by Cimg $=\boldsymbol{f}\left(K_{1}\right.$, image $)=\boldsymbol{f}\left(Q_{\mathrm{b}}{ }^{k_{x}}\right.$, image $)$.

(7) Bob deciphers the image by Dimg $=\boldsymbol{f}^{-1}\left(K_{1}\right.$, image $)=\boldsymbol{f}^{-1}\left(Q_{\mathrm{b}}{ }^{k_{x}}\right.$, image $)$.

\section{B. Confusion}

The confusion (also called discretization, permutation) stage shuffles the pixels in the image. It is an important technique used in image encryption and information hiding. Scientists have been conducting investigations in this field for a long time. Typical approaches to confusion include Arnold permutation [13], Hilbert permutation [14], Kolmogorov flows [15], baker map [16], Knight's tour problem [16-17], standard map [18], etc.
These existing techniques imply that an effective algorithm for confusion should follow the principles as below:

(1) Transform T must be 1-1 map. This is the primate premise for confusion. Only by following this rule can we make sure each pixel of cipher image will not lose its original information, so that it can be completely decrypted.

(2) T must disorganise the correlated positions of the plain-image as much as possible, such that the cipherimage cannot be directly seen by the human eyes or be guessed the information of the original image.

(3) $\mathrm{T}$ can rapidly disorganise the correlated positions of the plain-image; this is an important measure of the efficiency of a confusion algorithm.

(4) The existing cryptosystems and the fast speed of computers make it difficult for any cipher-image to resist the brute-force attack. For the security of the encryption algorithm, key space is needed to be as large as possible.

As a result, based on the discretization property of ergodic matrix, we propose a confusion scheme that goes by the following steps:

First, given $Q_{\mathrm{b}}{ }^{k x}$ is the key to encrypt the image, if the size of $Q_{\mathrm{b}}{ }^{k x}$ is smaller than that of the image, Alice calculates the power of $Q_{\mathrm{b}}$ by the following algorithm:

(1) $\mathrm{H}$ and $\mathrm{W}$ are the height and width of the image respectively;

(2) $\mathrm{N}$ is the number of the elements of the key $Q_{\mathrm{b}}{ }^{k x}$;

(3) $\mathrm{n}$ rounds the elements of $\mathrm{H} \times \mathrm{W} / \mathrm{N}$ to the nearest integers greater than or equal to $\mathrm{H} \times \mathrm{W} / \mathrm{N}$;

(4) ArrayB stores the result of $n$ matrices that Alice calculates;

(5) for $\mathrm{i}=1$ to $\mathrm{n}$

(6) the i-th row of ArrayB stores the result of $\operatorname{Power}\left(Q_{\mathrm{b}}{ }^{k x}, Q_{\mathrm{b}}{ }^{k x}(\mathrm{i})\right)$;

(7) end

(8) $\mathrm{IX}=\operatorname{sort}($ ArrayB) returns an array of indices after sorting ArrayB.

Then Alice discretizes the image according to IX. For example, if $\operatorname{IX}(i)=37$, the $i$-th pixel of the image is put to the $37^{\text {th }}$ position.

The experimental result for this discretization algorithm is shown in Figure 2.

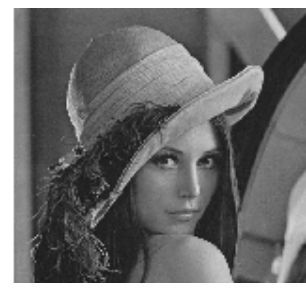

(a) Original Lena image

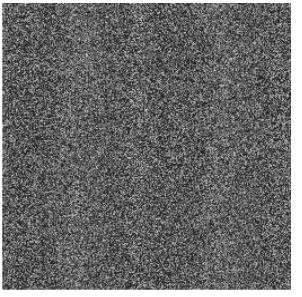

(b) Permuted image
Figure 2: Comparison of images after 1-round of permutation

It is easy to prove that this discretization is 1-1 map, which satisfies the $1^{\text {st }}$ principle above. Furthermore, the permuted image disorganizes the plain-image as much as possible so that it is hard to recognize, which satisfies the $2^{\text {nd }}$ principle. 


\section{Diffusion}

Shannon suggested employing diffusion and confusion in the cryptosystem [24]. The diffusion stage is necessary because an attacker can break the system by comparing a plain-image and corresponding cipher-image to discover useful information. For the purpose of diffusion, an explicit function which uses the ergodic matrix and the "XOR plus mod" [18] operation will spread out the influence of a single pixel, which is from the plain-image, over many cipher image pixels. This is detailed below.

Respectively choose the first element from $Q_{\mathrm{b}}{ }^{k x}$ and the plain-image as initial value.

$Q_{\mathrm{b}}{ }^{k x}$ is designed as $M(k)$ and is XOR-ed with the values of currently operated pixel (from the plain-image) and previously operated pixel (from the cipher-image), according to formula (1) below:

$C(\mathrm{k})=M(k) \oplus\{[I(k)+M(k)] \bmod C$ Level $\} \oplus C(k-1)$

where $I(k)$ is the currently operated pixel, CLevel is the colour level (for the image used to test, CLevel=256) of the image and $C(k-1)$ is the previously output pixel of the cipher-image. Bob may inverse the transform of the above formula as formula (2).

$I(k)=\{[M(k) \oplus C(\mathrm{k}) \oplus C(k-1)+C$ Level $-I(k)\} \bmod$ CLevel. (2)

We can see that the corner pixel $C(1)$ (namely, the position $(1,1)$ in the image) is not diffused at all under this algorithm. Besides, one pixel change in the image may not alter the cipher-image much, especially if the change is in the last pixel (namely the position is $(512,512))$. Hence another diffusion stages from the last pixel in the image is needed.

The experimental result for this discretization algorithm is shown in Figure 3.
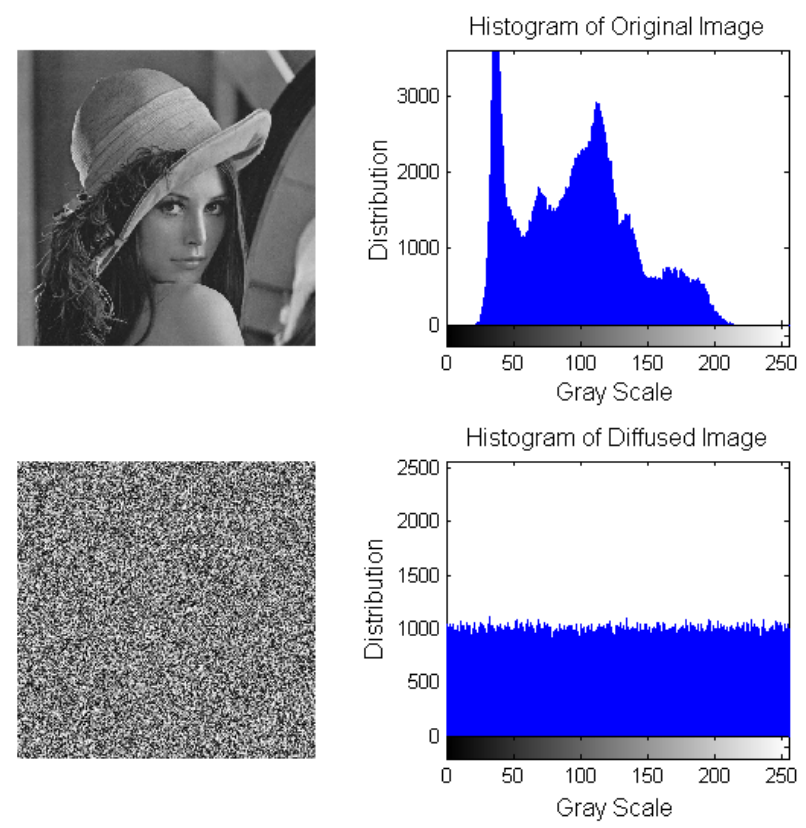

Figure 3: Comparison of images after 1-round of diffusion

\section{SECURITY ANALYSIS}

All tests in this paper are conducted on the $512 \times 512$ Lena image with 8-bit gray scale. The diffusion round is 2 , whilst the confusion round is 1 .

\section{A. Key space analysis}

The key space of any cryptosystem should be satisfactorily large enough to resist brute-force attack. In this proposed public-key based image encryption algorithm, key $\left(x_{\mathrm{a}}, y_{\mathrm{a}}=\boldsymbol{f}\left(x_{\mathrm{a}}, k_{\mathrm{a}}\right)\right.$ and $\left(x_{\mathrm{b}}, y_{\mathrm{b}}=\boldsymbol{f}\left(x_{\mathrm{b}}, k_{\mathrm{b}}\right)\right)$ are solely employed for encryption and decryption. Hence the key space primarily lies on the size of ergodic matrix (meaning unclear). For an $n \times n$ ergodic matrix over finite field $\mathbb{F}^{q}$, the number of non-equivalent matrices is calculated by fomula (3) [23]:

$$
\prod_{i=0}^{n-1} \frac{q^{n}-q^{i}}{n\left(q^{n}-1\right)}=\left(q^{n}-q\right)\left(q^{n}-q^{2}\right) \cdots\left(q^{n}-q^{n-1}\right) / n
$$

In the experiments we utilize a $50 \times 50$ ergodic matrix over finite field $\mathbb{F}^{256}$, thus the key space is

$$
\prod_{i=0}^{50-1} \frac{256^{50}-256^{i}}{50 \times\left(256^{50}-1\right)}
$$

This is quite large thus it is sufficient for practical use and can resist all kinds of brute force attack.

The reason for the immense key space in the proposed scheme is that the session keys are $n \times n$ matrices, of which the range of each element is $[0,255]$.

From Lemma 1, any ergodic matrix $Q \in \mathbb{F}_{n \times n}^{q}$ can be denoted by an $n$-vector over $\mathbb{F}^{q}$. Thus the size of the matrix $Q \in \mathbb{F}_{50 \times 50}^{256}$ used in the experiment can be reduced to 50 bytes from 2500 bytes.

\section{B. Statistical analysis}

\section{(1) Histogram analysis}

We can see from Figure 3 that after 1-round diffusion, the histogram is fairly uniform and does not reveal any statistical information of the plain-image.

\section{(2) Correlation of two adjacent pixels}

The high correlation of adjacent pixels is fragile to resist statistical cryptanalysis. As a result, a secure encryption scheme, which can eliminate the correlation between adjacent image pixels, is needed. Hence to calculate the correlation of two adjacent pixels, formula (4) is carried out:

$$
=\frac{\operatorname{cov}(x, y)}{\sqrt{\sum_{i=1}^{R}\left(x_{i}-E(x)\right)^{2}} \times \sqrt{\sum_{i=1}^{R}\left(y_{i}-E(y)\right)^{2}}}
$$

where $E(x)=\frac{1}{R} \sum_{i=1}^{R} x, R$ is the number of pairs of the adjacent pixels selected in the test. This formulate indicates $-1 \leq \operatorname{cov}(x, y) \leq 1$, which means with $\operatorname{cov}(x, y)$ getting closer to 0 , the correlations of two adjacent pixels will be less.

The experiment was divided into 10 groups and each group has 10,000 pairs of pixels, i.e. $R=10,000$. The correlation distributions of two adjacent pixels in the cipher-image are tested respectively in horizontal, vertical and diagonal. Table 1 shows the results of the correlation coefficients of our proposed algorithm. 
The mean correlation coefficients for our algorithm and the comparisons with other algorithms are listed in
Table 2.

TABLE 1. CORRELATION COEFFICIENTS OF TWO ADJACENT PIXELS IN THE CIPHER-IMAGE

\begin{tabular}{|l|l|l|l|l|l|l|l|l|l|l|}
\hline Horizontal & -0.00563 & 0.00021 & -0.01433 & -0.00089 & -0.0018 & -0.00207 & -0.00388 & 0.010665 & 0.013546 & 0.011906 \\
\hline Vertical & 0.0033113 & 0.015407 & 0.0071656 & -0.000164 & -0.000174 & -0.008530 & -0.004694 & -0.003341 & 0.0001274 & 0.010477 \\
\hline Diagonal & -0.004935 & 0.009473 & 0.0011877 & -0.008290 & 0.016918 & 0.0018506 & -0.005332 & 0.01446 & 0.0040661 & -0.01054 \\
\hline
\end{tabular}

TABLE 2. CORRELATION COEFFICIENTS COMPARED IN TWO ALGORITHMS

\begin{tabular}{|c|c|c|c|c|c|}
\hline & Plain-image & $\begin{array}{c}\text { Cipher-image } \\
\text { (Proposed, mean value) }\end{array}$ & $\begin{array}{c}\text { Cipher-image } \\
\text { (Yang et al. [9]) }\end{array}$ & $\begin{array}{c}\text { Cipher-image } \\
(\text { Ye G. [26]) }\end{array}$ & $\begin{array}{c}\text { Arnold method } \\
([26])\end{array}$ \\
\hline Horizontal & 0.98024213 & 0.006493 & 0.002097 & -0.0134 \\
\hline Vertical & 0.97533157 & 0.005339 & -0.016187 & 0.0012 \\
\hline Diagonal & 0.96573878 & 0.007705 & 0.017805 & 0.0398 & -0.0793 \\
\hline Average & 0.97377083 & 0.006512 & 0.01203 & 0.0181 \\
\hline
\end{tabular}

Both experiments utilized $512 \times 512$ Lena image with 256 gray scales. It is easy to see that the result of our algorithm is much closer to 0 . This indicates that our algorithm has effectively removed the correlation of adjacent pixels in the plain-image, thus it is better for image confusion and diffusion.

Test results for correlation of adjacent pixels are shown in Figure 4:

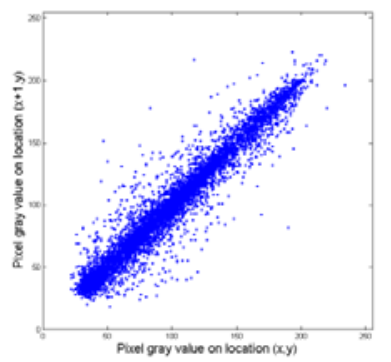

(a) The plain-image

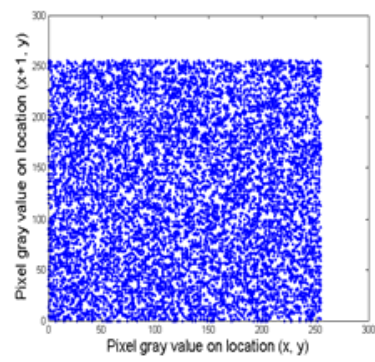

(b) The cipher-image
Figure 4: correlation of two horizontally adjacent pixels in (a) and (b)
Results imply that it is very difficult to deduce secret key from cipher-image when it is attacked by knowplaintext attacks or chosen-plaintext attacks.

\section{(3) Entropy analysis}

Entropy is a scalar value representing the entropy of a greyscale image. It is a statistical measure of randomness that can be used to characterize the texture of the input image. Entropy of an image is defined as:

$$
\mathrm{E}=\sum_{i=0}^{n} p\left(x_{i}\right) \log _{2} p\left(x_{i}\right)
$$

where $p$ contains the histogram counts returned from imhist.

The ideal value of entropy of a cipher-image should be 8. If it is less than this value, there will be some certain predictability that threatens the security.

Table 3 lists the mean entropy values obtained for different original image and the ciphered ones. The obtained results are much closed to the theoretical value. This means that information leakage after 1-round permutation and 2-round diffusion is so tiny that it can be neglected.

TABLE 3. ENTROPY VALUES FOR DIFFERENT ORIGINAL IMAGE AND THE CIPHERED ONES

\begin{tabular}{|l|c|c|c|}
\hline Original image & Cipher-image & $\begin{array}{c}\text { Entropy of the Original- } \\
\text { image }\end{array}$ & Entropy of the Cipher-image \\
\hline & a & & \\
\hline
\end{tabular}




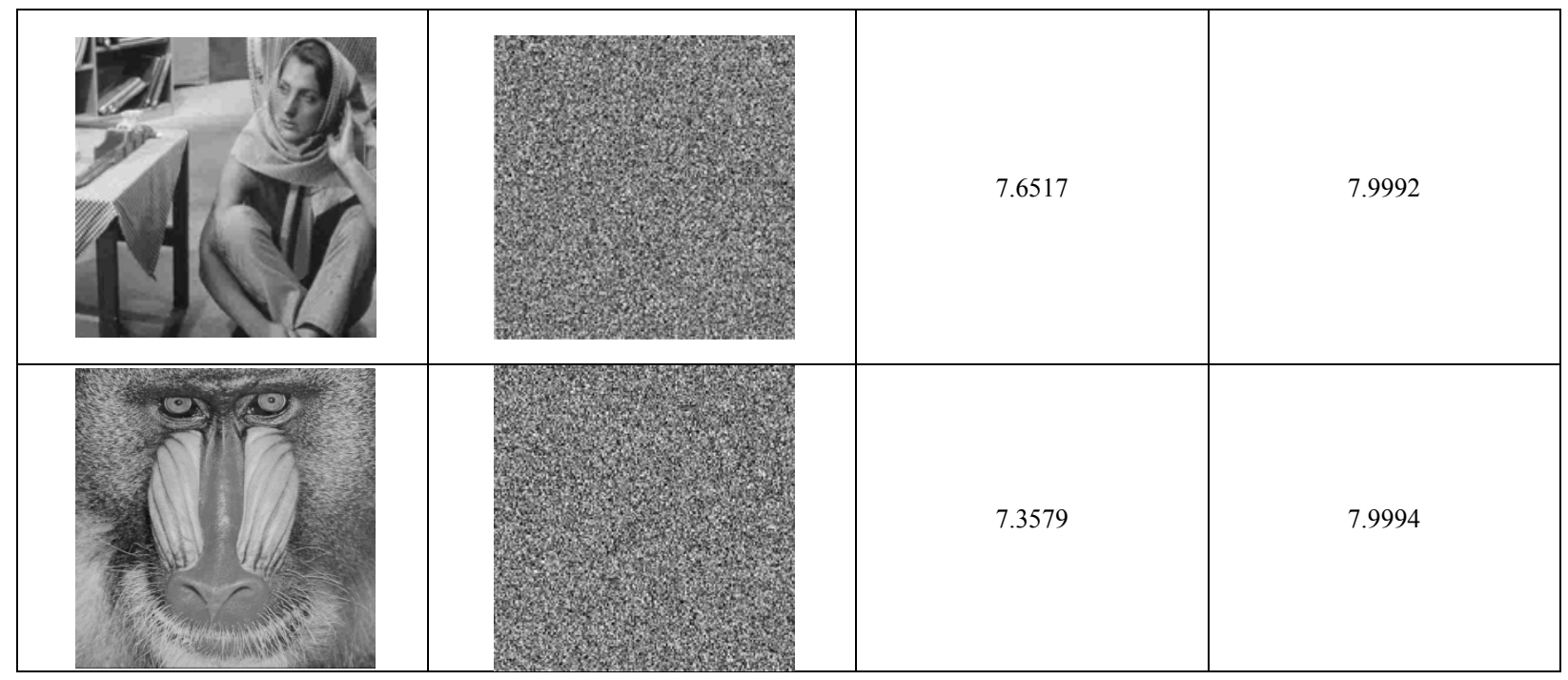

TABLE 4. 10 GROUPS OF NPCR AND UACI OF THE PROPOSED ALGORITHM

\begin{tabular}{|l|l|l|l|l|l|l|l|l|l|l|}
\hline NPCR & 0.99714 & 0.99544 & 0.99828 & 0.99829 & 0.99342 & 0.99809 & 0.99823 & 0.99812 & 0.99306 & 0.99802 \\
\hline UACI & 0.34382 & 0.33469 & 0.35326 & 0.31945 & 0.33498 & 0.3532 & 0.33637 & 0.34685 & 0.33266 & 0.34643 \\
\hline
\end{tabular}

TABLE 5. 10 GROUPS OF NPCR AND UACI OF DIFFERENT KEYS

\begin{tabular}{|l|l|l|l|l|l|l|l|l|l|l|}
\hline NPCR & 0.99586 & 0.99604 & 0.99608 & 0.99607 & 0.99613 & 0.99612 & 0.99613 & 0.99615 & 0.99608 & 0.99607 \\
\hline UACI & 0.33447 & 0.33457 & 0.33458 & 0.33446 & 0.33449 & 0.33455 & 0.33453 & 0.33460 & 0.33451 & 0.33453 \\
\hline
\end{tabular}

TABLE 6. CORRELATION COEFFICIENTS WITH KEY QBKX INCREASED BY 1 AT DIFFERENT POSITIONS

\begin{tabular}{|c|c|c|c|c|c|}
\hline position & $\begin{array}{c}\text { left-top } \\
(1,1)\end{array}$ & $\begin{array}{c}\text { right-top } \\
(1,50)\end{array}$ & $\begin{array}{c}\text { left-bottom } \\
(50,1)\end{array}$ & $\begin{array}{c}\text { right-bottom } \\
(50,50)\end{array}$ & $\begin{array}{c}\text { centre } \\
(25,1)\end{array}$ \\
\hline Correlation Coefficient & -0.00217286 & 0.004266592 & 0.0026746637 & -0.000910659 & -0.00149085 \\
\hline
\end{tabular}

\section{Sensitivity-based attack}

An algorithm for encrypting an image should be robust enough to resist sensitivity-based attack. This means the cryptosystem should have high key sensitivity and plaintext sensitivity [19]. Further, a tiny change, even a single pixel being modified by one bit, in the key or in the original image, causes a great difference in the cipherimage. These properties make it difficult for diverse sensitivity-based (chosen plaintext, or differential) attacks to break the system.

\section{(1) plain-image sensitivity}

The plain-image sensitivity of the cryptosystem is largely infected by the keys that Alice and Bob are holding. Here, two common measures are used to test the system: NPCR (Number of Pixels Change Rate) and UACI (Unified Average Changing Intensity). They are defined as formula (6) [7]:

$$
\left\{\begin{array}{c}
\text { NPCR }=\frac{\sum_{k=1}^{P} D_{k}(\text { pixelValue }(i, j))}{H \times W} \times 100 \% \\
\mathrm{UACI}=\frac{\sum_{k=1}^{P}\left|C_{1}^{k}(i, j)-C_{2}^{k}(i, j)\right|}{H \times W \times 255} \times 100 \%
\end{array}\right.
$$

where $H$ and $W$ are the height and the width of encrypted image. $\mathrm{D}_{k}($ pixelValue $(i, j))$ is determined by the following rule: if the pixel value of $\mathrm{C}_{1}(i, j)=\mathrm{C}_{2}(i, j)$ then $\mathrm{D}($ pixelValue $(\mathrm{i}, \mathrm{j}))=0$; otherwise $\quad \mathrm{D}($ pixelValue $(\mathrm{i}, \mathrm{j}))=1$. NPCR calculates the average intensity of different pixel numbers between two cipher-images with only one or more pixels changed.

UACI measures the percentage of pixel value differences between the two cipher-images.

Experiments have been carried out on the influence of a one-bit change on a 256 gray-scale Lena image of size $512 \times 512$, on the proposed cryptosystem. To get higher accuracy, we divided the experiment into 10 groups, in each of which was arbitrary chosen 1,000 pixels (of different positions, the corresponding pixel value only increased by 1) from the cipher-image.

The values of NPCR and UACI for our algorithm are listed in Table 4.

We can see from Table 4 that the lowest NPCR and UACI is 0.99306 and 0.31945 , respectively; the highest NPCR and UACI is 0.99829 and 0.35326, respectively; the average of these two measures' value is 0.996809 and 0.34071, respectively. While the NPCR and UACI of the proposed, Yang et al.'s cryptosystems is 0.996185 and 0.334795 , respectively [9].

Results show that the average performance of the proposed scheme only requires 1-round confusion stage and 2-round diffusion stage to slightly exceed the performance of the scheme introduced by Yang et al. [9], Furthermore, our experiment was tested on the slight change of different pixels and a large number of cases $(10 \times 1,000)$, while Yang et al. tested their system with 1 case. Thus our results are more precise. 


\section{(2) Key sensitivity}

The key sensitivity of our proposed scheme benefits from the matrix key $\mathrm{K}_{1}$. To evaluate, the key value is increased by 1 at a random position. The results are depicted in Figure 5, which shows that even a difference as small as one value incremented by 1 , will result in an incorrectly decrypted image.

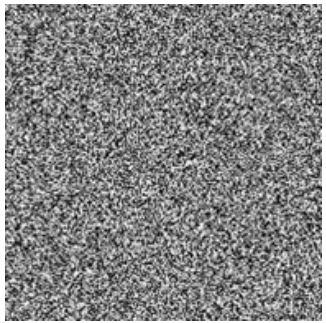

(a)

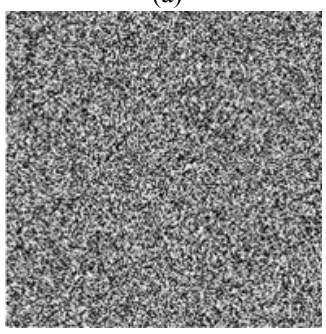

(c)

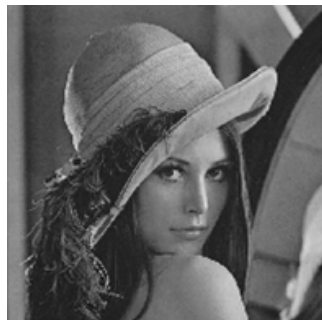

(b)

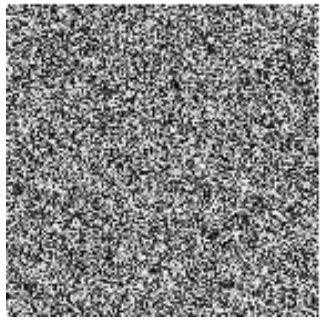

(d)
Figure 6: Key sensitivity test: (a) cipher-image using the key $\mathrm{K}_{1}$, (b) image encrypted with $K_{1}$ (c) cipher-image using the left-top value of matrix key increased by 1 , (b) image encrypted with $\mathrm{K}_{1}$.

It is difficult to compare the cipher-image by merely observing these images. So for comparison, NPCR and UACI are calculated between the two cipher-images using a different key which is increased by 1 at a random position.

For this calculation, we used the same formula given in (4) except that in this test is the values of corresponding pixels in the two cipher-images to be compared. The result is shown in Table 5.

We can see from Table 5 that the average of two cipher-images is 0.996073 and 0.334529 , respectively. To get more precise results, another test, which calculates the correlation between the corresponding pixels between the two cipher-images, is implemented. For this calculation, we used the same formula given in (3) except that in this test, it is the values of corresponding pixels in the two cipher-images that are compared in Table 6.

Results show that, for example, the cipher-image by the original key $\mathrm{K}_{1}$ has $99.78 \%$ of difference (this is better than the result from the algorithm proposed by Ismail et al. [20], which was 99.59\%) from the one encrypted by the key $\mathrm{K}_{1}(1,1)+1$ in terms of pixel grey scale values, although there is only one tiny difference in the two keys.

\section{PERformance eVAluation}

Apart from security considerations, other issues concerning the image cryptosystem, such as the performance speed, also play a significant role. The performance of an algorithm is affected by many conditions such as: the programmer's skill; what kind of programme language he is using; the performance of the computer he performs tests on; and how many bits the operation system and the software have.

All the experiments in this paper were carried out with MATLAB programming language as it is strong and readable in the handling of images and matrices. The implementation was done on a personal computer with a 3.20 GHz Core2Duo processor and 2 GB main memory, running with the Windows XP 32-bit operation system.

The encryption procedure consists of three main stages: Calculation of the public key $Q_{\mathrm{b}}$, confusion and diffusion. Table 7 shows the CPU running time when the image was encrypted with the key $Q_{\mathrm{b}}{ }^{k x}$ in Matlab.

TABLE 7. THE RUNNING TIME (MS) OF CPU WITH THE KEY K $\mathrm{K}_{1}$ IN MATLAB

\begin{tabular}{|c|c|c|c|}
\hline$k_{x}\left(\right.$ of $\left.Q_{\mathrm{b}}{ }^{k x}\right)$ & $\begin{array}{c}\text { Confusion } \\
\text { stage }\end{array}$ & $\begin{array}{c}\text { Diffusion } \\
\text { stage }\end{array}$ & Calcualtion of $Q_{\mathrm{b}}{ }^{k x}$ \\
\hline 1 & 15.625 & 242.375 & 0.022 \\
\hline 100 & 15.625 & 234.750 & 15.625 \\
\hline 10,000 & 15.625 & 242.375 & 15.625 \\
\hline 1000,000 & 15.625 & 223.875 & 31.250 \\
\hline $100,000,000$ & 15.625 & 242.375 & 46.875 \\
\hline $10,000,000,000$ & 15.625 & 215.625 & 62.500 \\
\hline $1,000,000,000,000$ & 15.625 & 250.000 & 62.500 \\
\hline
\end{tabular}

Experiment results indicate that it takes on average 200 to 400 milliseconds for the encryption and decryption stage respectively. It is obvious that the speed of confusion stage and the calculation of the power of $Q_{\mathrm{b}}$ is quite fast whilst the diffusion stage consumes a significant amount of time. However, in fact, the diffusion stage is largely the same as that proposed by Yang et al.[9] and Ismail et al. [20]. The reason for the difference is that the use of the bitwise XOR operation results in a longer delay in Matlab than in low level languages. For example, a loop that uses the operation and repeats $512^{2}$ times takes less than 1 millisecond to complete in $\mathrm{C}$, whereas the same loop in Matlab takes 1.5 seconds.

To compare, we also test in $\mathrm{C}$. Table 8 shows the $\mathrm{CPU}$ running time while using $\mathrm{C}$ to realize our algorithm.

TABLE 8. THE RUNNING TIME (MS) OF CPU WITH THE KEY K 1 IN C

\begin{tabular}{|c|c|c|c|}
\hline$k_{x}\left(\right.$ of $\left.Q_{\mathrm{b}}{ }^{k x}\right)$ & $\begin{array}{c}\text { Confusion } \\
\text { stage }\end{array}$ & $\begin{array}{c}\text { Diffusion } \\
\text { stage }\end{array}$ & $\begin{array}{c}\text { Calculation time of } \\
Q_{\mathrm{b}}{ }^{k x}\end{array}$ \\
\hline 1 & 1.969 & 2.047 & 0.0266 \\
\hline 100 & 1.984 & 2.063 & 0.0313 \\
\hline 10,000 & 1.969 & 2.031 & 0.0312 \\
\hline 1000,000 & 1.953 & 2.047 & 0.0328 \\
\hline $100,000,000$ & 1.922 & 2.063 & 0.0328 \\
\hline $10,000,000,000$ & 1.953 & 2.063 & 0.0343 \\
\hline $1,000,000,000,000$ & 1.954 & 2.031 & 0.0360 \\
\hline $100,000,000,000,000$ & 1.937 & 2.047 & 0.0500 \\
\hline $256^{50}-1$ & 1.945 & 2.041 & 1787.6 \\
\hline
\end{tabular}

Table 8 shows the running time of confusion and diffusion is quite stable (it is 1.9 to 2.0 milliseconds and 2.0 to 2.1 milliseconds, respectively). The running time of the power of a $50 \times 50$ matrix is even much faster than that of confusion and diffusion. Even the matrix $Q_{\mathrm{b}}$ to the 
power of $256^{50}-1$, which we thought will consume much time, only takes 1.7876 seconds.

Therefore, compared to the algorithms proposed by Yang and Ismail in 2010, according to the performance evaluation, the sensitivity and statistical analysis, our proposed algorithm is more suitable for higher security purposes and is also suitable for network transmission.

\section{CONCLUSION AND FUTURE WORK}

A hybrid-key based image encryption and authentication scheme is proposed in this paper. Ergodic matrix, which is almost uniformly distributed, plays a central role in the encryption/decryption process. It is demonstrated that an ergodic matrix $Q \in \mathbb{F}_{50 \times 50}^{256}$ can be employed to completely shuffle and diffuse the original image and has an immense key space of at least $3.08 \times 10^{5898}$. With this key space, the scheme is robust enough for the cryptosystem to resist the bruteforce attack. Normally, 1-round diffusion and 1-round permutation is enough for the encryption, but it is vulnerable to differential attack, which, however, is ineffective if a tiny change in the original image (or session key) will cause a great difference in the cipherimage. As a result of this, we have applied one more round of diffusion. It is shown that, in the 2-round diffusion and 1-round permutation scheme, either a single pixel is modified by only one bit in the original image, or only one element of the session key is increased by 1 , a significant difference will occur in the cipher-image.

Compared with the existing chaotic cryptosystems [3-9, $15,16,19]$, the experimental tests conducted in this paper demonstrate more optimistic results: the change rate in the number of pixels and unified average changing intensity are both higher, whilst the correlation coefficient is lower.

We can see from Table 8 that the diffusion stage consumes much of the encryption time. How can we achieve a better trade-off between the computing complexity and the security? Is any way that the diffusion stage can be reduced to one round? These are the issues we shall concern ourselves with in our future work.

\section{REFERENCES}

[1] Acharya, B., S. K. Panigrahy, et al. (2009). "Image Encryption Using Advanced Hill Cipher Algorithm." International Journal of Recent Trends in Engineering 1(1): 663-667.

[2] Öztürk, I. and I. Sogukpınar (2005). "Analysis and Comparison of Image Encryption Algorithms." World Academy of Science, Engineering and Technology 3: 26-30.

[3] Zhang, L., X. Liao, et al. (2005). "An image encrypton approach based on chaotic maps." Chaos, Solitions and Fractals 24: 759-765.

[4] Fridrich, J. (1998). "Symmetric ciphers based on two-dimensional chaotic maps." International Journal of Bifurcation and Chaos 8(6): 1259-1284.
[5] Giesl, J., L. Behal, et al. (2009). "Improving Chaos Image Encryption Speed." International Journal of Future Communication and Networking 2(3): 23-36.

[6] Miao, Y., G. Chen, et al. (2004). "A novel fast image encryption scheme based on 3D chaotic baker maps." International Journal of Bifurcation and Chaos 14(10): 3613-3624.

[7] Chen, G., Y. Mao, et al. (2004). "A symmetric image encryption scheme based on 3D chaotic cat maps." Chaos, Solitions and Fractals 21: 749-761.

[8] Guan, Z., F. Huang, et al. (2005). "Chaos-based image encryption algorithm." Physics Letters $A$ 346(1-3): 153-157.

[9] Yang, H., K. Wong, et al. (2010). "A fast image encryption and authentication scheme based on chaotic maps." Communications in Nonlinear Science and Numerical Simulation 15(11): 35073517.

[10]Zhao, Y., L. Wang, et al. (2004). "InformationExchange Using the Ergodic Matrices in GF(2)." Proceedings of the 2nd ACM symposium on Information, Computer and Communications Security ACNS 2004: 347-349.

[11]Zhou, X., J. Ma, et al. (2010). "BMQE system: an MQ euqations system based on ergodic matrix." Proceedings of the International Conference on Security and Cryptography: 431-435.

[12]Diffie, W. and M. E. Hellman (1976). "New Directions in Cryptography." IEEE Transactions on Information Theory 22(6): 644-654.

[13]Runger, G. C. and M. L. Eaton (1992). "Most powerful invariant permutation tests." Journal of Multivariate Analysis 42(2): 202-209.

[14]Lin, S.-Y., C.-S. Chen, et al. (2003). "Tensor product formulation for Hilbert space-filling curves." Proceedings of Parallel Processing 2003, Kaohsiung.

[15]Josef, S. (2000). "Secure digital watermark generation based on chaotic Kolmogorov flows." Security and Watermarking of Multimedia Content II, Proceedings of SPIE, 2000 3971: 306-313.

[16] Miyamoto, M., K. Tanaka, et al. (1999). "Truncated baker transformation and its extension to image encryption." Proceedings of SPIE on Advanced Materials and Optical System for Chemical 3858: 13-25.

[17] Parberry, I. (1996). "Scalability of a neural network for the Knight's tour problem." Neurocomputing 12(1,15): 19-33.

[18] Parberry, I. (1997). "An efficient algorithm for the Knight's tour problem." Discrete Applied Mathematics 73(3,21): 251-260.

[19]Lian, S., J. Sun, et al. (2005). "A block cipher based on a suitable use of the chaotic standard map." Chaos, Solitions and Fractals 26: 177-129.

[20] Ismail, I. A., M. Amin, et al. (2010). "A digital image encryption algorithm based a composition of two chaotic logisitc maps." International Journal of Network Security 11(1): 1-10. 
[21] Anonymous (1998, 10/09/98). "Introduction to public-key cryptography." [online]. Available at http://docs.sun.com/source/816-6154-10/.

[22] Menezes, A. J., P. C. v. Oorschot, et al. (1996). Handbook of Applied Cryptography. United States, CRC Press: 816.

[23]Zhao, Y., S. Pei, et al. (2007). "Using the Ergodic Matrices over Finite Field to Construct the Dynamic Encryptor." Journal of Chinese Computer Systems 2007(11): 2010-2014.

[24] Shannon, C. (1949). "Communication theory of secrecy systems." Bell System Technical Journal 28(4): 656-715.

[25] Jormakka, J. (2004). "Symmetric and asymmetric cryptography overview." from http://www.netlab.tkk.fi/opetus/s38153/k2003/Lectur es/g33symm_asymm_crypto.pdf.

[26] Ye Guodong. (2010). "Image scrambling encryption algorithm of pixel bit based on chaos map." Pattern Recognition Letters 31 :347-354

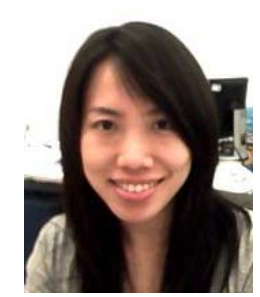

Xiaoyi Zhou, Hainan, China, 1979.

She received a M.Sc. degree in Applied Computer Science from Jilin University, Jilin, China in 2005. Currently, she is a Ph.D Research Scholar of the University of Greenwich (London, UK) and Hainan University (Hainan, China). Her research interests include Cryptography and Temporal Logic.

She is a lector in the College of Information Sciences \& Technology, Hainan University, China.

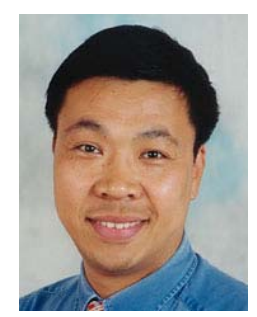

Jixin Ma, Henan, China, 1963.

$\mathrm{He}$ received a $\mathrm{PhD}$ degree in Computer Science from the University of Greenwich, London, UK. His research interests include Artificial Intelligence and Graph Matching.

$\mathrm{He}$ is a member of: the American Association of Artificial Intelligence; the China-Britain Technology and Trade Association (committee member); the World Scientific and Engineering Society; and the UK Temporal Reasoning, Artificial Intelligence and Logic Group.

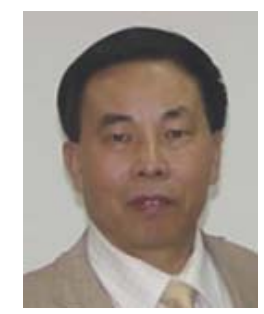

Wencai Du, Jiangsu, China, 1953.

$\mathrm{He}$ received a $\mathrm{PhD}$ degree in Computer Science from the University of South Australia, Australia in 1999. His research interests include E-business and network communication.

$\mathrm{He}$ is a Professor \& Dean in the College of Information Sciences \& Technology, Hainan University, China. $\mathrm{He}$ is also a member of the China High Vocation and Technique Education Committee, a President of Electronics Society of Hainan Province, China and a Vice-President of Software Association of Hainan Province, China.

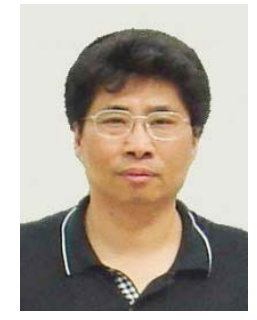

Yongzhe Zhao, Beijing, China, 1961.

$\mathrm{He}$ received a M.Sc. degree in Computer Science from Tianjin University, China in 1987. His research interests include Cryptography and Information Security.

$\mathrm{He}$ is a professor in Computer Science and Technology School, Jilin University, China. 